\title{
PROPIEDADES FÍSICAS DEL GRANO Y CALIDAD DE LOS GRUPOS RACIALES DE MAÍCES NATIVOS (CRIOLLOS) DE MÉXICO
}

\author{
PHYSICAL KERNEL PROPERTIES AND QUALITY OF RACIAL GROUPS OF MAIZE \\ LANDRACES OF MÉXICO
}

\author{
Juan de D. Figueroa Cárdenas ${ }^{1 \star}$, David E. Narváez González ${ }^{2}$, Araceli Mauricio Sánchez ${ }^{1,2}$, Suketoshi Taba ${ }^{3}$, \\ Marcela Gaytán Martínez ${ }^{1}$, José J. Véles Medina ${ }^{1}$, Froylán Rincón Sánchez ${ }^{4}$ y Flavio Aragón Cuevas ${ }^{5}$
}

\begin{abstract}
${ }^{1}$ Centro de Investigación y de Estudios Avanzados, Unidad Querétaro. Libramiento Norponiente 2000. 76230, Fracc. Real de Juriquilla, Querétaro, Qro. ${ }^{2}$ Universidad Autónoma de Querétaro. DIPA. Cerro de las Campanas s/n. 76010, Querétaro, Qro. ${ }^{3}$ Centro Internacional de Mejoramiento de Maíz y Trigo (actualmente jubilado). km 45 carr. México-Veracruz. 56130, El Batán, Texcoco, Edo. de México. ${ }^{4}$ Universidad Autónoma Agraria Antonio Narro. Calz Antonio Narro 1923. 25315, Buenavista, Saltillo, Coah. ${ }^{5}$ Instituto Nacional de Investigaciones Forestales, Agrícolas y Pecuarias, Campo experimental Valles Centrales Oaxaca. Melchor Ocampo 7. 68200, Santo Domingo, Barrio Bajo, Etla, Oax.
\end{abstract}

*Autor para correspondencia (jigueroa@qro.cinvestav.mx)

\section{RESUMEN}

La calidad del maíz (Zea mays L.) ha sido el principal criterio de selección utilizado por nuestros ancestros para su mejoramiento. De hecho, gran parte del maíz que consume nuestra población ha estado sujeto durante siglos a la selección por sabor, aroma y textura en diferentes productos. Sin embargo, las clasificaciones y los registros de razas, híbridos y variedades se han hecho desde el punto de vista agronómico, citológico y taxonómico, y existen pocos estudios sobre la evaluación de sus propiedades físicas y de calidad. El presente trabajo de revisión tiene por objetivo analizar los resultados que se han publicado en el contexto de la calidad de los maíces nativos (razas) de México, y compararlos con las clasificaciones agronómicas de las razas. Los datos analizados muestran que las clasificaciones agronómicas de los grupos raciales del maíz en México, aunque importantes para el manejo en programas de mejoramiento, no presentaron relación directa con los aspectos de calidad para tortilla y otros usos. La dureza de grano o el índice de flotación fueron las características más relacionadas con el desempeño de las razas en el procesamiento y calidad de los productos y pudiera ser importante incorporarlas a los sistemas de clasificación.

Palabras clave: Zea mays, razas de maíz, calidad tortillera, propiedades físicas, uso industrial.

\section{SUMMARY}

Maize (Zea mays L.) selection for kernel quality has been the main basis for improvement for our ancestors. Most maize consumed today has undergone centuries of selection by flavor, odor and texture in various products such as tortillas. However, classification and registry of landraces and hybrids is based on agronomical, cytological and taxonomical characteristics; very few studies have evaluated physical properties and quality of kernels. This review explores published research related to corn quality of Mexican maize landraces and contrasts those results to the agronomical classification of Mexican maize landraces. Data showed that agronomic classification of Mexican landraces, although important for genetic breeding programs, is not directly related with quality features for tortilla production or other final uses. Kernel hardness and flotation index were traits highly related to race performance and quality of the maize products, so it might be important to incorporate them in classification systems.

Index words: Zea mays, maize landraces, races, tortilla quality, physical properties, industrial use.

\section{INTRODUCCIÓN}

Gran parte de la diversidad genética del maíz (Zea mays L.) está concentrada en el continente Americano y principalmente en México. El maíz es consumido en esas zonas geográficas en múltiples formas, como tortillas, arepas, pinoles, atoles, tostadas, botanas tamales, elotes y otros muchos alimentos (Figueroa et al., 2005). La elaboración de estos productos requiere de granos con características específicas, las cuales son identificadas con base en características externas, tales como color, tamaño y dureza. Adicionalmente, el grano de maíz puede ser empleado en muchos tipos de industrias, como la textil, química, de cosméticos y alimentaria (Rooney et al., 2003), de las cuales la industria alimentaría es más importante que las otras debido a que existen tantas maneras de elaborar los productos como la variabilidad genética lo permite. Por ejemplo, el almidón de maíz puede ser usado en la industria de alimentos como espesante, estabilizador, agente formador de gel, y como agente encapsulador (Schoch, 1970).

En México la mayor parte de los agricultores que cultivan maíz lo destinan al autoconsumo. Estos agricultores utilizan todavía variedades locales y generalmente ellos mismos se encargan de producir su semilla. Se estima que debido a esta situación la superficie sembrada con variedades mejoradas e híbridos en México es de apenas $20 \%$ aproximadamente (Morris y López-Pereira, 2000). Debido a lo anterior, es todavía posible obtener variedades criollas de maíz propias de la región y evaluar su potencial de industrialización.

El presente trabajo tiene por objetivo presentar los resultados que se han publicado sobre la calidad de los maíces criollos, así como usar dichos criterios de calidad para responder 
a las siguientes preguntas: ¿existe alguna relación entre las clasificaciones agronómicas de los grupos raciales de maíz y la calidad?, ¿cómo explicar la calidad del grano y sus productos?, ¿cómo afectan las propiedades físicas del grano de maíz a la calidad y el rendimiento industrial?

\section{Clasificación racial del maíz}

Una de las primeras clasificaciones del maíz (Zea mays L.) fue la realizada por Sturtevant (1899), quien propuso seis grupos principales (dentado, cristalino, dulce, harinoso, reventón, ceroso y tunicado), cinco de los cuales se basaron en la composición del endospermo de los granos. Posteriormente, Anderson y Cutler (1942) consideraron que la clasificación de Sturtevant era muy artificial porque estos grupos no eran indicativos de relaciones naturales entre ellos, por lo que propusieron una clasificación basada en la constitución genética total que incluyera características de espiga, mazorca y grano.

En la misma década en que Anderson y Cutler (1942) propusieron su clasificación de los maíces de México, Centro y Sudamérica y parte de los Estados Unidos, varios investigadores (Longley, 1938, 1941; Mangelsdorf y Cameron, 1942; y Brown, 1949) describieron las variaciones citológicas del maíz y su relación con la diversidad regional y varietal. Por su parte, Wellhausen et al. (1952) reportaron el origen, las características y la distribución de las razas de maíz en México, con base en el estudio de aproximadamente 2000 variedades, en cuanto a su distribución geográfica, características vegetativas de la planta, de la espiga y de la mazorca, así como en características fisiológicas, genéticas y citológicas.

Sin embargo, con excepción de la primera las demás clasificaciones omitieron los aspectos de calidad del grano, aspectos que probablemente fueron la base de la selección por su uso. Tal omisión posiblemente se debió a la complejidad en la definición de calidad. El limitado conocimiento del uso y calidad del material genético ha repercutido en pérdida de material valioso en los programas de fitomejoramiento genético.

\section{Selección por calidad del grano}

Como se mencionó, la clasificación de las razas de maíz en México se ha hecho desde los puntos de vista botánico, genético, agronómico, citológico y taxonómico (Wellhausen et al., 1952; Hernández X. y Alanís, 1970), con énfasis en descriptores fenotípicos de la mazorca o grano (Wellhausen et al., 1952). Hasta hoy pocos trabajos han abordado los caracteres físicos del grano y su relación con la calidad de la tortilla (Mauricio et al., 2004; Figueroa et al., 2005; Vázquez et al., 2010), y menos aún los aspectos microestructurales y su relación con el uso final y la calidad del grano (NarváezGonzález et al., 2007a) y su potencial industrial (Figueroa et al., 2005; Nárvaez-González et al., 2006a y 2006b; NárvaezGonzález et al., 2007b; Aragón et al., 2012).

Excepto por el autoconsumo en las comunidades rurales que han privilegiado la calidad sobre el rendimiento agronómico, el sistema actual de comercialización de maíz heredado de la ya extinta Comisión Nacional de Subsistencia Popular que controló los precios de 1962 a 1999, favorece el abasto sin considerar los aspectos de calidad intrínseca del maíz y otros granos. Es a partir de la apertura de mercados internacionales, como el Tratado de Libre Comercio de América del Norte en 1994 que condujo a la desaparición de aranceles al maíz en 2008 y la globalización comercial, que se ha retomado la calidad debido a que los consumidores y procesadores de maíz están buscando los apropiados para sus procesos y productos.

Los granos con alto valor agregado son los que presentan características especiales de calidad que aumentan el beneficio para el usuario (Gómez et al., 2006a; 2006b). En este grupo se encuentran los siguientes maíces: el maíz azul, como fuente de pigmentos (antocianidinas) y antioxidantes naturales, muy apreciado para hacer tortillas, tostadas y botanas (Salinas et al., 2007; De la Parra et al., 2007); el maíz blanco, con antioxidantes naturales (Del Pozo et al., 2006; Ruiz-Torres et al., 2008); al maíz de endospermo duro, para la industria de la molienda seca, por sus propiedades de baja absorción de agua y aceite para producir botanas (Rooney y Serna-Saldívar, 2003); el maíz palomero, para elaborar rosetas; el maíz de endospermo semiduro, para la industria de las harinas nixtamalizadas para tortillas (Aragón et al., 2012); los maíces con alto porcentaje de germen, porque incrementan el rendimiento de aceite y reducen el uso de este ingrediente en los alimentos balanceados (Aragón et al., 2012); los maíces poli-embriónicos con alto contenido de aceite, para harinas con menor retrogradación (Cruz-Requena et al., 2011); el maíz con alto contenido de almidón y el de bajo contenido de fitatos (Layrisse et al., 2000); el maíz con bajo porcentaje de fisuras internas, para el secado a temperaturas bajas; el maíz no transgénico NOGMO (organismo no modificado genéticamente, por sus siglas en inglés) (Johnson y May, 2003; Turrent et al., 2009), para utilizarse en productos alimenticios de exportación; el maíz nutricionalmente acrecentado, con alto contenido de proteína; el maíz orgánico, libre de pesticidas después de la cosecha; el maíz ceroso y el maíz blanco (Gómez et al., 2006a; 2006b).

\section{Calidad de grano en las razas de maíz en México}

La importancia del maíz en México, especialmente como parte de la alimentación de su población, ha sido 
documentada ampliamente (Randolph, 1952; Kato y McClintock, 1981; Figueroa y Aguilar, 1997). Actualmente se reconocen 346 razas de maíz, de las cuales 59 se encuentran en México (Kato et al., 2009) y aún son sembradas en gran variedad de regiones agroecológicas que van desde el nivel de mar hasta $3000 \mathrm{~m}$ de altitud (Hernández-Casillas, 1999). El Cuadro 1 muestra los grupos raciales y las razas pertenecientes a cada grupo.

Según Mauricio et al. (2004), entre las propiedades importantes para la clasificación del uso alimentario del maíz en México están el tamaño del grano, su gravedad específica y su dureza, así como su capacidad de absorción de agua y rendimiento de masa, rendimiento de tortilla, la pérdida de peso durante la cocción de la tortilla y la resistencia al corte de la tortilla. El color del grano de maíz varía ampliamente entre genotipos, y aunque no se considera una propiedad importante para su uso alimentario, influye considerablemente en la preferencia del consumidor (Mauricio et al., 2004; Aragón et al., 2012).
Además, Mauricio et al. (2004) reportaron diferencias significativas $(\mathrm{P} \leq 0.01)$ entre grupos raciales para todas las características del grano, así como entre razas dentro de grupos (Cuadro 2); si bien no encontraron diferencias significativas entre grupos raciales para variables de rendimiento y de calidad tortillera, pero sí las detectaron entre razas dentro de grupos en cuanto a capacidad de absorción de agua, pérdida de peso y resistencia al corte $(\mathrm{P} \leq 0.05)$.

Algunos autores indican que la calidad final de la tortilla y otros productos obtenidos por el método tradicional de nixtamalización dependen de varios factores, como tipo de maíz, tiempo de cocimiento, porcentaje de cal, tiempo de reposo y pérdida de sólidos, por lo que se necesita una metodología apropiada para lograr la calidad deseada (Gaytán et al., 2006; Castillo et al., 2009; Fernández-Muñoz et al., 2010). Unos autores propusieron el cocimiento óhmico para predecir la calidad del grano (Gaytán et al., 2012); en dicho cocimiento se hace pasar una corriente eléctrica a través del maíz molido con agua y cal, mezcla que funciona como resistencia eléctrica (efecto Joule), para lograr un

Cuadro 1. Clasificación de las razas mexicanas de maíz.

Grupo racial

Razas Indígenas Antiguas

Exóticas Precolombinas

Razas Mestizas-Prehistóricas

Modernas Incipientes

Razas No bien Definidas

Razas no clasificadas en alguno de los grupos propuestos
Nombre de la raza

Palomero Toluqueño (1); Arrocillo Amarillo (1); Chapalote (1); Nal-Tel (1).

Cacahuacintle (1); Harinoso de Ocho (1): Sub-Raza Elotes Occidentales $\left(3,^{\dagger}\right)$; Olotón; Maíz Dulce (1).

Cónico (1); Reventador (1); Tabloncillo (1); Tehua (1); Tepecintle (1); Comiteco (1); Jala (1); Zapalote Chico (1); Zapalote Grande (1); Pepitilla (1); Olotillo (1): Subraza: Dzit-Bacal (3); Tuxpeño (1); Vandeño (1).

Chalqueño (1); Celaya (1); Cónico Norteño (1); Bolita (1).

Conejo ( ${ }^{\dagger}$ ); Mushito (6); Complejo Serrano de Jalisco (4); Zamorano Amarillo (6); Maíz Blando de Sonora (6); Onaveño (4); Dulcillo del Noroeste (6).

Azul (2); Apachito (2); Tablilla de Ocho (2); Gordo (2); Tuxpeño Norteño (5); Bofo (2); Onaveño (4); Coscomatepec (5, 6); San Juan (7); Carmen (7); Cónico Mushito $\left(^{\dagger}\right)$; Tablón-Cónico Norteño $\left(^{\dagger}\right)$; Conejo Vandeño $\left(^{\dagger}\right)$; Blandito (6); Blandito Gordo $\left(^{\dagger}\right)$; Cristalino de Chihuahua (5); Cristalino de Chihuahua-Tablón $\left(^{\dagger}\right)$; AzulBofo $\left({ }^{\dagger}\right)$; Chalqueño-Elotes Cónicos $\left(^{\dagger}\right)$; Cónico-Norteño-Bofo ( ${ }^{\dagger}$; Ancho-Olotillo $\left({ }^{\dagger}\right)$; Olotón-Bolita $\left(^{\dagger}\right)$; Cónico-Bolita $\left({ }^{\dagger}\right)$; Cónico-Cacahuacintle $\left({ }^{\dagger}\right)$; Tunicado $\left(^{\dagger}\right)$; Maizón (8); Xmehenal $\left({ }^{\dagger \dagger}\right)$; Tablón-Perla (5); Lady Finger $\left({ }^{\dagger \dagger}\right)$; Clavillo $\left({ }^{\dagger \dagger}\right)$; Fasciado $\left(^{\dagger \dagger}\right)$; Ratón (5); Palomero de Chihuahua (5); Chatino Maizón (8); Mixeño (8); Choapaneco (8); Mixteco (8); Serrano Mixe (8). Motozinteco (5, 6); Elotero de Sinaloa $(5,6)$.

1. Razas definidas por Wellhausen et al. (1952); 2. Razas definidas por Hernández X. y Alanís (1970); 3. Subrazas, según Wellhausen et al. (1952); 4. Razas No Bien Definidas, según Wellhausen et al. (1952); 5. Razas descritas por Ortega-Paczka (1985) Variedades y razas mexicanas de maíz y su evaluación en cruzamientos con líneas de clima templado como material de partida para fitomejoramiento. Tesis de grado Ph.D. Leningrado, URSS. 22 p.; 6. Razas descritas por Sánchez-González (1989) Relationships among the Mexican races of maize. Unpublished Ph.D. dissertation, North Carolina State University, Raleigh. 187 p.; 7. Razas descritas por Ortega-Paczka y Barajas (1994); 8. Razas descritas por Benz (1986); ${ }^{\dagger}$ Maíz proveniente de la cruza de dos razas. ${ }^{\dagger \dagger}$ Razas no clasificadas en alguno de los grupos propuestos. 
Cuadro 2. Comparación de grupos raciales de maíz por sus dimensiones de grano.

\begin{tabular}{lccccccccc}
\hline Grupo racial & $\begin{array}{c}\text { Largo } \\
(\mathrm{mm})\end{array}$ & $\begin{array}{c}\text { Ancho } \\
(\mathrm{mm})\end{array}$ & $\begin{array}{c}\text { Espesor } \\
(\mathrm{mm})\end{array}$ & PMG $(\mathrm{g})$ & Dureza $(\mathrm{N})$ & GS $\left(\mathrm{g} \mathrm{mL}^{-1}\right)$ & $\mathrm{CAA}$ & PP & RT \\
\hline Indígenas Antiguas & $10.1 \mathrm{~b}$ & $6.7 \mathrm{~d}$ & $5.0 \mathrm{ab}$ & $186.6 \mathrm{c}$ & $12.8 \mathrm{a}$ & $1.2767 \mathrm{a}$ & $1.08 \mathrm{~b}$ & $29.74 \mathrm{a}$ & $1.46 \mathrm{a}$ \\
Exóticas Precolombinas & $10.2 \mathrm{~b}$ & $10.0 \mathrm{a}$ & $5.5 \mathrm{a}$ & $343.7 \mathrm{a}$ & $9.4 \mathrm{~b}$ & $1.1257 \mathrm{c}$ & $1.19 \mathrm{a}$ & $31.30 \mathrm{a}$ & $1.51 \mathrm{a}$ \\
Mestizas-Prehistóricas & $11.4 \mathrm{ab}$ & $9.0 \mathrm{ab}$ & $4.9 \mathrm{ab}$ & $306.7 \mathrm{ab}$ & $11.0 \mathrm{ab}$ & $1.1973 \mathrm{~b}$ & $1.45 \mathrm{a}$ & $31.50 \mathrm{a}$ & $1.45 \mathrm{a}$ \\
Modernas Incipientes & $12.8 \mathrm{a}$ & $7.5 \mathrm{~cd}$ & $4.8 \mathrm{ab}$ & $330.0 \mathrm{ab}$ & $12.9 \mathrm{a}$ & $1.2133 \mathrm{~b}$ & $1.40 \mathrm{a}$ & $32.98 \mathrm{a}$ & $1.40 \mathrm{a}$ \\
No Bien Definidas & $10.9 \mathrm{~b}$ & $8.4 \mathrm{bc}$ & $4.5 \mathrm{~b}$ & $260.4 \mathrm{~b}$ & $11.8 \mathrm{ab}$ & $1.1760 \mathrm{bc}$ & $1.43 \mathrm{a}$ & $33.04 \mathrm{a}$ & $1.43 \mathrm{a}$ \\
\hline
\end{tabular}

Medias con letras iguales en la misma columna son estadísticamente iguales (Tukey, 0.05). GS = gravedad específica; CAA = capacidad de absorción de agua, en $\mathrm{kg}$ de agua por kg de harina; $\mathrm{PP}=$ pérdida de peso durante el cocimiento de la tortilla, en $\%$ del peso inicial; $\mathrm{RT}=$ rendimiento de tortilla, en $\mathrm{kg}$ de tortilla por kg de harina. $P M G=$ peso de mil granos.

grado deseado de cocimiento (Gaytán et al., 2012), lo cual representa una ventaja sobre el cocimiento del proceso tradicional de nixtamalización (Gaytán et al., 2012). Aun así, la variación en la calidad nixtamalera y en características del grano puede ser útil para la clasificación y discriminación de accesiones de maíz.

Razas Indígenas Antiguas. Este grupo incluye a las siguientes razas: Palomero Toluqueño, Arrocillo Amarillo, Chapalote y Nal-Tel (Cuadro 1). Las cuatro razas tuvieron un desarrollo independiente en diferentes localidades y ambientes, pero con ciertas características en común, como el endospermo duro típico del maíz palomero y de los maíces de mazorcas pequeñas (Mauricio et al., 2004). Todas estas razas, como su ancestro, producen granos reventadores y por ello se usan para elaborar palomitas o rosetas (Cuadro $3)$.

Existen dos subrazas del Palomero Toluqueño: Palomero Poblano con mazorcas cónicas y grano puntiagudo de textura suave, a diferencia del Palomero Toluqueño que tiene endospermo córneo, diferencia que permite suponer una relación intermedia entre Palomero Toluqueño y Pepitilla; y el Palomero Jalisciense cuyas mazorcas son menos cónicas que el Palomero Poblano (Wellhausen et al., 1952; Romero et al., 2005).

Con estos maíces también se pueden elaborar tortillas, pero éstas no presentan texturas suaves (Romero et al., 2005). No obstante, Vázquez et al. ( 2010) han reportado usos para tortilla en las razas Arrocillo y Palomero Toluqueño del Atiplano y Valle del Mezquital de Hidalgo, mediante variaciones en procedimientos que permiten modificar la calidad de nixtamal, masa y tortilla, aunque la mayoría de estos granos mantiene valores bajos de humedad y de rendimiento de tortilla; por ejemplo, la raza Palomero Toluqueño presenta uno de los más altos porcentajes de pérdida de sólidos en el grupo estudiado.

Razas Exóticas Precolombinas. Las razas que pertene- cen a este grupo son: Cacahuacintle, Harinoso de Ocho, Olotón y Maíz Dulce (Cuadro 1). Se caracterizan por tener granos largos con endospermo harinoso de color blanco y textura suave (Cuadro 3). Se usan para preparación de platillos mexicanos tradicionales, como pozoles, elotes (mazorcas inmaduras), atoles (bebidas espesas) y tortillas (Mauricio et al., 2004; Vázquez et al., 2010). Cacahuacintle es una raza sui generis para elaborar pozoles y elotes debido a su rápido cocimiento, textura suave y firme del producto, además de alto rendimiento de pozole. Elotes occidentales es una subraza de color morado del altiplano de Jalisco relacionada con la raza Harinoso de Ocho (Wellhausen et al., 1952). Esta subraza es muy apreciada por su sabor dulce en elotes asados, elotes cocidos, atoles coloreados, pinole (granos tostados y molidos) y también para pozole (Figueroa et al., 2005; Ron et al., 2006). La raza Maíz Dulce se emplea en pinole, ponteduro (bolas de granos tostados y esponjados unidos con jarabe), sopas y caldos (Figueroa et al., 2005; Ron et al., 2006).

Razas Mestizas-Prehistóricas. Estas razas se formaron del entrecruzamiento de las Indígenas Antiguas y las Exóticas Precolombinas, con introgresión del teocintle, especialmente Z. mays L. ssp. parviglumis y Z. mays L. ssp. mexicana (Wellhausen et al., 1952; Aragón et al., 2006). Se denominan Prehistóricas porque no existe evidencia histórica de su origen y no es seguro de que todas sean precolombinas, ya que varias de éstas pudieron haberse originado como consecuencia de las migraciones de los primeros colonizadores. Hasta ahora sólo se han reconocido trece razas de este grupo: Cónico, Reventador, Tabloncillo, Tehua, Tepecintle, Comiteco, Jala, Zapalote Chico, Zapalote Grande, Pepitilla, Olotillo, Tuxpeño y Vandeño.

Según Mauricio et al. (2004), este grupo racial está conformado por una mayoría de razas de buena calidad tortillera, como Cónico, Jala, Olotillo, Tepecintle, Tuxpeño Norteño, Vandeño y Zapalote Chico (Cuadro 3). La buena calidad tortillera de las razas Pepitilla y Tuxpeño ha sido confirmada por varios autores (Vázquez et al., 1990; Vázquez et al., 2010; 


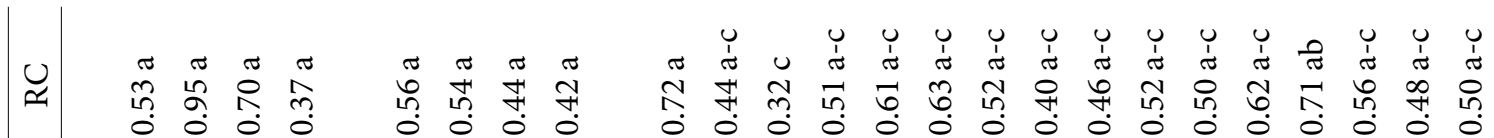

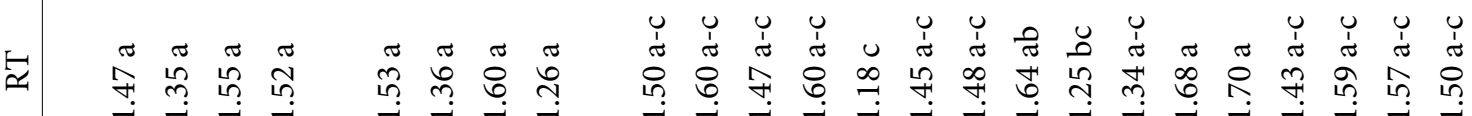

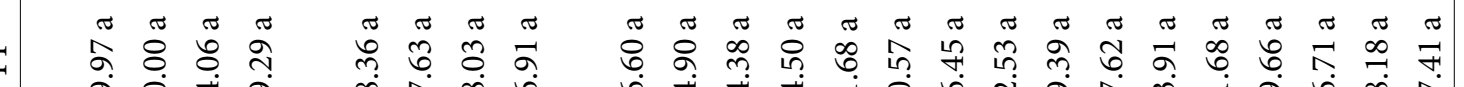
సें

\ति

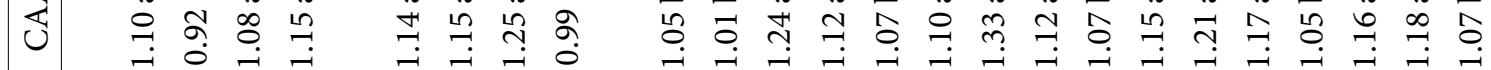

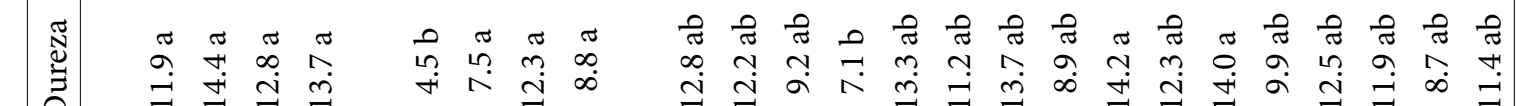

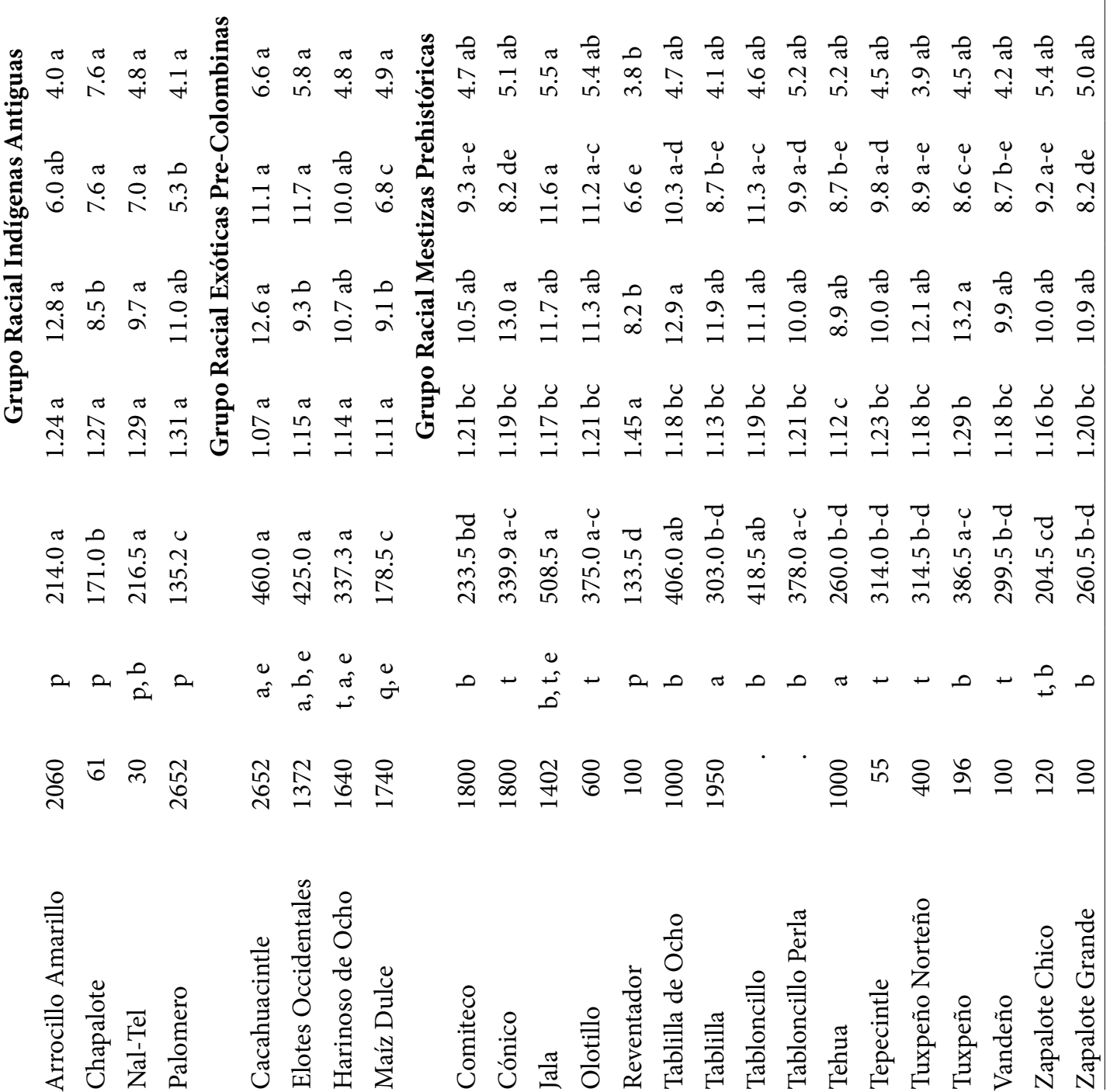




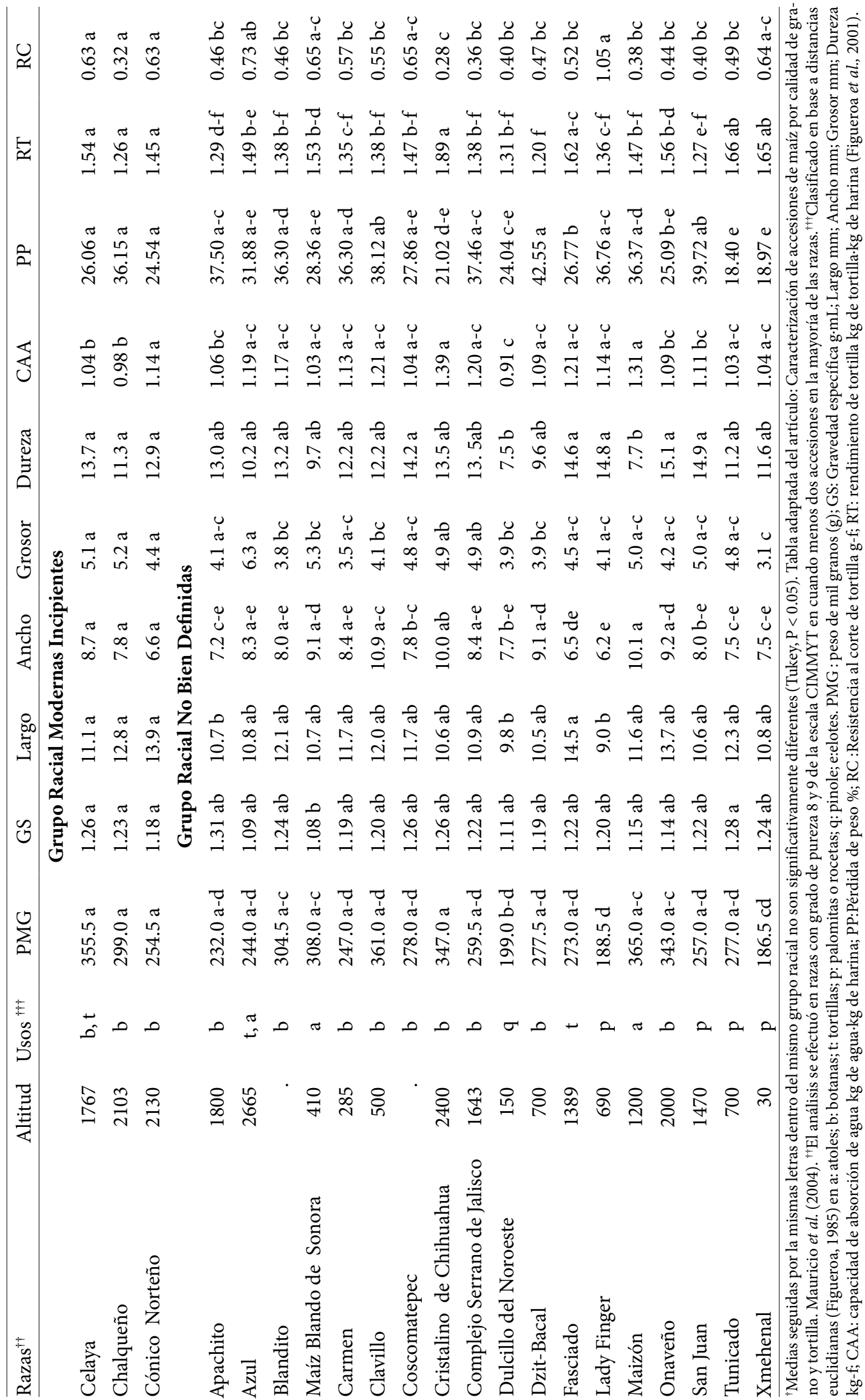


Antuna et al., 2008). El elevado porcentaje de germen (12.0 a $15.1 \%$ ) en el grano de la raza Pepitilla (Aragón et al., 2006), le confiere mayor suavidad a la tortilla. Algunas razas del grupo Mestizas Prehistóricas pueden tener otros usos, como el caso de las razas Comiteco, Tehua y Reventador que también se usan para elaborar palomitas. Por su baja dureza de grano y alto contenido de azúcares simples, la raza Jala se ha propuesto para el consumo en elotes (Valdivia-Bernal et al., 2010).

Razas Modernas Incipientes. Estas son razas que se han desarrollado desde la época de la conquista: Chalqueño, Celaya, Cónico Norteño, Tablita y Bolita. La raza Celaya (Cuadro 3), presenta características de grano apropiadas para elaborar tortillas y botanas (Mauricio et al., 2004). Las razas Chalqueño, Cónico Norteño, Bolita y Celaya sirven para elaborar tortilla y harinas (Vázquez et al., 2010). Por su parte, Gómez et al. (2006a, 2006b) reportaron el potencial de industrialización de 42 accesiones criollas de maíz cultivadas en siete municipios del Estado de Hidalgo, identificadas como pertenecientes a las razas Cónico, Chalqueño y Celaya; las tortillas elaboradas con los maíces criollos de Hidalgo fueron más suaves que las reportadas por Vázquez et al. (2010).

Razas No Bien Definidas. Son razas de colectas hechas por Wellhausen et al. (1952), quienes no contaron con suficientes datos para clasificarlas dentro de un grupo genealógico más homogéneo. Entre éstas se encuentran: Conejo, Muschito, Complejo Serrano de Jalisco, Zamorano Amarillo, Maíz Blando de Sonora, Onaveño, Dulcillo del Noroeste, Cristalino de Chihuahua, Elotero de Sinaloa y Azul (Cuadros 1 y 3). Algunas razas de este grupo tienen características notables, como Conejo que es apreciada por su precocidad por producir los primeros elotes y granos en el ciclo agrícola. Por su parte, Aragón et al. (2006) reportaron que su grano es de muy alto contenido de germen (13.5 a $19.6 \%$ ), lo que le confiere ventajas como fuente de energía en alimentos balanceados.

La raza Azul de la Sierra Tarahumara, con buen rendimiento y calidad de tortilla, se utiliza también en pinole, atole y en la extracción de antocianinas, y se exporta a Estados Unidos para la elaboración de botanas como totopos y frituras. Apachito es de la Sierra Tarahumara y presenta un grano rosado utilizado para pinole, elotes, atoles, tortillas y tesgüino (bebida alcohólica). Cristalino de Chihuahua, que se derivó de Apachito, tiene grano de endospermo cristalino y semi-cristalino, y es además una de las razas más precoces de México, y se utiliza en elotes asados y hervidos, y en tortillas. En el grano de Dulcillo de Noreste, raza probablemente derivada de las razas Maíz Dulce y Reventador (Wellhausen et al., 1952), la conversión de azúcares a almidón durante el llenado del grano no se completa normal- mente, por lo que presenta mayor contenido de azúcares libres y un sabor especial a sus productos; se utiliza en pinole, elotes y esquites (CONABIO, 2010). Estos usos reportados por diversos autores coinciden con las estimaciones del uso potencial descrito en el Cuadro 3, obtenido a partir de sus propiedades físicas y de procesamiento del grano.

\section{Dureza de grano y calidad de tortilla}

Las características de grano y de calidad tortillera anotadas en los Cuadros 2 y 3 permiten clasificar a las accesiones de maíz entre y dentro de grupos raciales. Mauricio et al. (2004) reportaron correlaciones significativas entre características del grano y la calidad tortillera, como entre la capacidad de absorción de agua y la gravedad específica $(\mathrm{r}=$ $-0.21 ; \mathrm{P} \leq 0.05)$ y con el ancho del grano $(\mathrm{r}=0.28 ; \mathrm{P} \leq 0.01)$, así como entre resistencia al corte, peso de mil granos $(\mathrm{r}=$ $0-0.23 ; \mathrm{P} \leq 0.05)$, y ancho del grano $(\mathrm{r}=-0.23 ; \mathrm{P} \leq 0.05)$.

Estos autores también reportaron que los granos aptos para producir atoles y pozoles se caracterizan por tener tamaño grande y valores bajos de dureza y gravedad específica, en tanto que los granos para tortillas tienen valores intermedios en tales características; en contraste, los granos para pinoles se caracterizan por ser cortos, anchos y gruesos, de baja dureza y gravedad específica. Los granos palomeros son pequeños, duros y de alta gravedad específica. Los granos empleados para botanas son los más largos y duros. Por ello los investigadores consideran que las características de mayor definición respecto al producto alimenticio obtenido, son dureza del grano, pérdida de peso y capacidad de absorción de agua, porque los valores altos de capacidad de absorción de agua se registran en los maíces para la producción de tortillas y atoles, mientras que los granos destinados a botanas tienen valores menores, y son aún menores en los maíces para palomitas y pinoles. Así pues, las características estudiadas de los granos de maíz, especialmente la dureza, se relacionan con el uso alimenticio al cual se destinan, y por lo mismo son útiles para definir la calidad industrial del grano respecto al producto que se desee elaborar.

En virtud de que la dureza y la gravedad específica son las características que definieron en mayor medida la calidad final de los maíces, tanto entre las razas y como dentro de los grupos raciales, Gaytán et al. (2006) estudiaron algunas características que pudieran estar influenciando la dureza en el grano de maíz, como: densidad del grano, tamaño del gránulo de almidón, grado de cristalinidad, tipo de endospermo (suave o duro), y proporción de pericarpio y germen. En varios reportes se han establecido las relaciones existentes entre la dureza del grano y el índice de flotación (Martínez y Lachance, 1979; Salinas et al., 1992; Norma Oficial Mexicana, 2002), amilosa y porcentaje de almidón (Dombrink-Kurtzman y Knutson, 1997), y tipo y cantidad 
de zeína (Dombrink-Kurtzman y Bietz, 1993; Lopes y Larkins, 1991; Moro et al., 1995).

Cabe precisar que el índice de flotación representa el número de granos de maíz que flotan en una solución de nitrato de sodio a una densidad de $1.25 \mathrm{~g} \mathrm{~mL}^{-1}$. Los granos duros presentan bajos índices de flotación, mientras que los maíces suaves presentan índices altos (Aragón et al., 2012). El Cuadro 2 muestra que en el grupo racial Indígenas Antiguas con densidad $1.2767 \mathrm{~g} \mathrm{~mL}^{-1} \mathrm{y}$ con alta dureza, sus granos no flotan en la solución de la prueba.

La dureza puede estar relacionada no solamente con las características químicas del grano, sino con las propiedades físicas. Ambas características afectan la manera en la cual las moléculas y partículas se ensamblan en el grano (Gaytán et al., 2006; Narváez-González et al., 2007a). Se han reportado correlaciones positivas de la dureza con densidad, tamaño del gránulo de almidón, y con el porcentaje de endospermo (Gaytán et al., 2006). Por otra parte, se encontró una correlación negativa entre el índice de flotación y dureza, y una positiva entre el índice de flotación y la cristalinidad y con los porcentajes de pericarpio y germen (Cuadro 4). El endospermo es el principal componente del grano de maíz y parece contribuir de manera importante en la dureza del grano, como se puede observar con la correlación positiva de este factor.

El arreglo estructural del gránulo de almidón dentro del grano es un factor que afecta más a la dureza que el grosor del pericarpio y que el contenido de fibra dentro de las células endospérmicas, o que la matriz proteica de los gránulos de almidón (Gaytán et al., 2006). La microscopía electrónica ha revelado que los granos de maíces duros presentan diferentes patrones que los granos de maíz suave (Figura 1).
En el endospermo del maíz suave los gránulos de almidón son principalmente esféricos y débilmente empaquetados en una matriz, mientras que los de maíz duro son poligonales y altamente empaquetados (Narváez-González et al., 2007a).

La dureza, como una característica de la textura de los granos de maíz, debe ser considerada como el resultado de la contribución de varias características que incluyen a los compuestos químicos individuales así como a sus interacciones, y a los arreglos moleculares dentro del gránulo de almidón y el grano de maíz. Otras características importantes son: densidad (gravedad específica), contenido de germen, porcentajes de endospermo y pericarpio, y tamaño del gránulo de almidón. A este respecto se ha observado que posiblemente lo más importante para definir la calidad son los aspectos intrínsecos o microestructurales del grano, influenciados éstos por las propiedades fisicoquímicas del mismo.

\section{CONCLUSIONES}

Las clasificaciones agronómicas de los grupos raciales del maíz en México, aunque importantes para el manejo en programas de mejoramiento, no presentaron una relación directa con los aspectos de calidad para tortilla y otros usos que destinan los agricultores. Se sugiere que a esas clasificaciones se le incorporen variables como la dureza del grano, para adaptarlas a las necesidades actuales del mercado y de los agricultores.

La calidad es el grupo de características que diferencian a una raza de otra, en un material o producto, y que marcan el grado de excelencia de la raza en el material o producto específico. Por tanto, la definición de calidad del maíz

Cuadro 4. Correlaciones entre características del grano y su dureza ${ }^{\dagger}$.

\begin{tabular}{|c|c|c|c|c|c|c|c|c|c|}
\hline \multirow{3}{*}{ Variable } & & IF & Dureza & Densidad & Gránulo & Cistalinidad & Endospermo & Pericarpio & Germen \\
\hline & Mín & 7.00 & 7.60 & 1.03 & 7.88 & 7.27 & 80.79 & 3.43 & 8.00 \\
\hline & Máx & 93.00 & 16.90 & 1.35 & 14.11 & 11.21 & 87.17 & 7.55 & 12.87 \\
\hline IF & & 1 & & & & & & & \\
\hline Dureza & & $-0.735^{\star}$ & 1 & & & & & & \\
\hline Densidad & & $-0.845^{\star}$ & $0.688^{\star}$ & 1 & & & & & \\
\hline Gránulo & & $-0.488^{\star}$ & $0.587^{\star}$ & $0.500^{*}$ & 1 & & & & \\
\hline Cristalinidad & & $0.753^{*}$ & $-0.440^{*}$ & $-0.530^{*}$ & $-0.330^{*}$ & 1 & & & \\
\hline Endospemo & & $-0.571^{\star}$ & $0.486^{\star}$ & $0.716^{\star}$ & $0.444^{\star}$ & -0.082 & 1 & & \\
\hline Pericarpio & & $0.413^{\star}$ & $-0.309^{*}$ & $-0.681^{\star}$ & -0.178 & 0.144 & $-0.647^{\star}$ & 1 & \\
\hline Germen & & $0.661^{*}$ & $-0.425^{\star}$ & $-0.574^{\star}$ & $-0.333^{\star}$ & $0.429^{*}$ & $-0.636^{*}$ & $0.332^{*}$ & 1 \\
\hline
\end{tabular}

IF = índice de flotación; Dureza (kg-f); Densidad ( $\left.\mathrm{g} \mathrm{mL}^{-1}\right)$; Tamaño del gránulo de almidón ( $\left.\mu \mathrm{m}\right)$; Endospermo (\%); Pericarpio (\%); Germen (\%). Las hileras: Min y Max indican el rango de las características del grano en la población evaluada. ${ }^{\star}$ Significativo a 0.05 nivel de probabilidad. ${ }^{\dagger}$ Adaptada de Gaytán et al. (2006). 


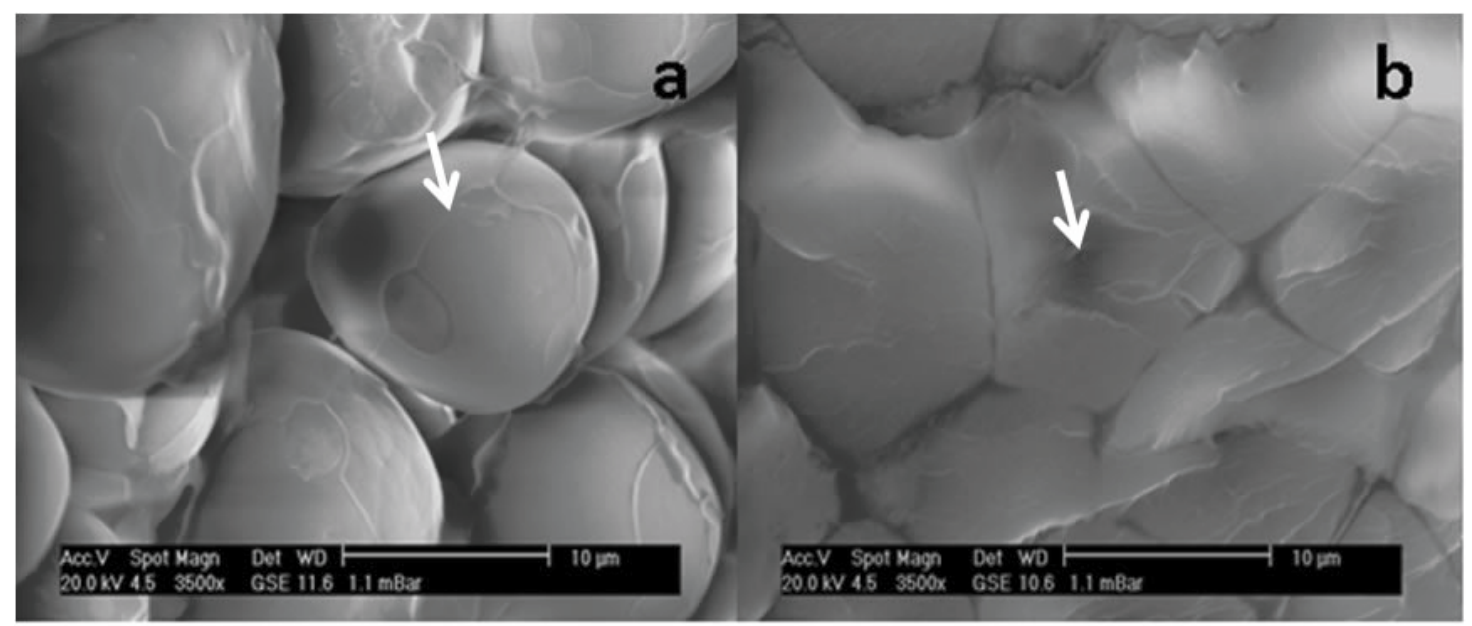

Figura 1. Microfotografía del gránulo de almidón a 3500 X: a) Forma esférica del gránulo de almidón en maíz de endospermo suave, b) Forma poligonal del gránulo de almidón en maíz de endospermo duro (Narváez-González et al., 2006a).

es una labor compleja que requiere conocer los métodos y características apropiadas a medir en cada material o producto a evaluar. Se sugiere la agrupación de razas por usos o productos, sin embargo, excepto para tortilla y usos industriales no alimenticios dónde se emplean híbridos, existe limitada información publicada de los métodos y parámetros de calidad para otros usos alimenticios de las razas mexicanas.

No se puede explicar la calidad con solamente un conjunto de variables, ya sea físicas del grano (tamaño, color, peso de mil granos), comúnmente utilizadas para la selección del material o producto. Sin embargo, la dureza de grano o el índice de flotación son mediciones indirectas de la microestructura, y se relacionan con el tipo y proporción de endospermo, así como con la humedad en el grano. Todos estos factores y sus inter-relaciones afectan el desempeño de las razas en el procesamiento y el rendimiento industrial.

\section{AGRADECIMIENTOS}

A "MASAGRO: Modernización sustentable de la agricultura tradicional", un programa de SAGARPA-CIMMYT, por su apoyo para la publicación de este manuscrito, y a N. Palacios Rojas por su revisión técnica.

\section{BIBLIOGRAFÍA}

Anderson E, H C Cutler (1942) Races of Zea mays: I. Their recognition and classification. Ann. Miss. Bot. Garden 29:69-89.

Antuna G O, S A Rodríguez-Herrera, G Arámbula-Villa, A Palomo-Gil, E Gutierréz-Arias, A Espinoza-Banda, E F Navarro-Orona, E Andrio-Enríquez (2008) Calidad nixtamalera y tortillera de maíces criollos de México. Rev. Fitotec. Mex. 31 (Esp. 3):23-27.

Aragón C F, J D C Figueroa, M Flores Z, M Gaytán M, M J Jéles (2012) Calidad Industrial de Maíces Nativos de la Sierra Sur de Oaxa- ca. Libro Técnico No. 15. Instituto Nacional de Investigaciones Forestales, Agrícolas y Pecuarias. Santo Domingo Barrio Bajo, Etla, Oaxaca, México. 249 p.

Aragón C F, S Taba, J M Hernández C, J D Figueroa C, A V Serrano, G F H Castro (2006) Catálogo de Maíces Criollos de Oaxaca. Libro Técnico No. 6. Instituto Nacional de Investigaciones Forestales, Agrícolas y Pecuarias. Santo Domingo Barrio Bajo, Etla, Oaxaca, México. 343 p.

Benz B F (1986) Taxonomy and evolution of Mexican maize. In: Studies in the Neolithic and Urban revolutions. L Manzanilla (ed). University of Colorado, Boulder, CO. pp:121-136.

Brown W L (1949) Numbers and distribution of chromosome knobs in United States maize. Genetics 34:524-536.

Castillo V K C, M L Ochoa A, J D Figueroa C, L Delgado E, J A Gallegos I, C J Morales (2009) Efecto de la concentración de hidróxido de calcio y tiempo de cocción del grano de maíz (Zea mays L.) nixtamalizado, sobre las características fisicoquímicas y reoló gicas del nixtamal. Arch. Latinoam. Nutr. 59:425-432.

CONABIO, Consejo Nacional de Biodiversidad (2010) Argumentación para conservar las razas de maíces nativos de México. Disponible en:http://www.biodiversidad.gob.mx/genes/pdf/proyecto/ Anexo6_ReunionesTalleres/Tabla\%20razas_marzo\%202010. pdf. Consultado Marzo 20, 2012.

Cruz-Requena M, R R Herrera, C N Aguilar González, J E Velázquez, M G Martínez, J D Figueroa C (2011) Alkaline cooking quality of polyembryonic and non-polyembryonic maize populations. Adv. J. Food Sci.Techn. 3:259-268.

De la Parra C, S O Serna S, L R Hai (2007) Effect of processing on the phytochemical profiles and antioxidant activity of corn for production of masa, tortillas, and tortilla chips. J. Agric. Food Chem. 55:4177-4183.

Del Pozo I D, C H Brenes, S O Serna S, T S Talcott (2006) Polyphenolic and antioxidant content of white and blue corn (Zea mays L.) products. Food Res. Intl. 39:696-703.

Dombrink-Kurtzman M A, J A Bietz (1993) Zein composition in hard and soft endosperm of maize. Cereal Chem. 70:105-108.

Dombrink-Kurtzman M A, C A Knutson (1997) A study of maize endosperm hardness in relation to amylose content and susceptibility to damage. Cereal Chem. 74:776-780.

Fernández-Muñoz J L, M E Rodríguez-García, J D C Figueroa, M LealPérez, H E Martínez-Flores, H Ortiz (2010) Evaluación de los cambios fisicoquímicos a diferentes tamaños de partícula de harinas de maíz nixtamalizado como función del tiempo de reposo. Superficies y Vacío 23:21-24

Figueroa J D C (1985) Métodos para Evaluar la Calidad Maltera de Cebada.Tema Didáctico Núm. 17. SARH-INIA. México, D.F. 115 p. 
Figueroa J D C, R Aguilar (1997) El origen del maíz. Avance y Perspectiva 16:91-98.

Figueroa J D C, M G Acero-Godínez, N L Vasco-Méndez, A LozanoGuzmán, L M Flores-Acosta, J González-Hernández (2001) Fortificación y evaluación de tortillas de nixtamal. Arch. Latinoam. Nutr. 51:293-302.

Figueroa J D C, R A Mauricio, S Taba, E Morales, A Mendoza-Gaytán, F Rincón-Sánchez, L M Reyes, J J Véles (2005) Kernel characteristics and tortilla making quality of maize accessions from Mexico, the Caribbean, and South and Central America. In: Latin American Maize Germplasm Conservation: Regeneration, in situ Conservation, Core Subsets, and Prebreeding. S Taba (ed). Proceedings of a Workshop held at CIMMYT, April 7-10, 2003. México, D.F.: CIMMYT. 71 p.

Gaytán M M, J D C Figueroa, M L Reyes-Vega, F Rincón-Sánchez, E Morales-Sánchez (2006) Microstructure of starch granule related to corn hardness. Rev. Fitotec. Mex. 29(Esp. 2):135-139.

Gaytán M M, J D C Figueroa, P-A Vázquez-Landaverde, E MoralesSánchez, H E Martínez-Flores, M L Reyes-Vega (2012) Caracterización fisicoquímica, funcional y química de harinas nixtamalizadas de maíz obtenidas por calentamiento óhmico y proceso tradicional. CyTA-J. Food 10:182-192.

Gómez R R, J D C Figueroa, M C Gayosso, E M Ramírez, A D F Hernández (2006a) Maíces criollos del Altiplano del Estado de Hidalgo. Libro SEP-Instituto Nacional del Derecho de Autor. Núm. de registro 03-2006-100614290800-01. $80 \mathrm{p}$.

Gómez R R, J D C Figueroa, M C Gayosso, E M Ramírez, A D F Hernández, L R Ledesma, T R Bilbao (2006b) Investigaciones sobre maíz en el Estado de Hidalgo, Mex. Compilación Libro SEP-Instituto Nacional del Derecho de Autor,. Núm de registro 03-2006-100614302100-01. $87 \mathrm{p}$

Hernández-Casillas J M (1999) La diversidad del maíz mexicano y su conservación. In: Memorias del 2do. Taller Nacional de Especialidades de Maíz. V J Espinoza, C J del Bosque (eds). Saltillo, Coah. pp:1-13.

Hernández X E, G Alanís (1970) Estudio morfológico de cinco nuevas razas de maíz de la Sierra Madre Occidental de México: Implicaciones fitogenéticas y fitogeográficas. Agrociencia 1:3-30.

Johnson L A, J B May (2003) Wet milling: The basis for corn biorefineries. In: Corn Chemistry and Technology. J P White, L A Johnson (eds). American Association of Cereal Chemists, Inc. $2^{\text {nd }} e d$. St Paul, MN. USA. pp:449-494.

Kato Y A, B McClintock (1981) The chromosome constitution of races of maize in north and middle America. Part Two. In: Chromosome Constitution of Races of Maize. Ed. Colegio de Postgraduados, Chapingo, México. pp:9-67.

Kato T A, C Mapes, L M Mera, J A Serratos, R A Bye (2009) Origen y Diversificación del Maíz: Una Revisión Analítica. Universidad Nacional Autónoma de México, Comisión Nacional para el Conocimiento y Uso de la Biodiversidad. México, D.F. 116 p.

Layrisse M, M N García-Casal, M A Barón, F Argüello, D Llovera, J Ramírez, I Leets, E Tropper (2000) New property of vitamin $\mathrm{A}$ and B-carotene on human iron absorption: effect on phytate and polyphenols as inhibitors of iron absorption. Arch. Latinoam. Nutr. 50:243-248.

Longley A E (1938) Cromosomes of maize from North American Indians. J. Agric. Res. 56:177-195.

Longley A E (1941) Chromosome morphology in maize and its relatives. Bot. Rev. 7: 263-289.

Lopes M A, B A Larkins (1991) Gamma-zein content is related to endosperm modification in quality protein maize. Crop Sci. 31:1655-1662.

Mangelsdorf P C, J W Cameron (1942) Western Guatemala. A Secondary Center of Origin of Cultivated Maize Varieties. Botanical Museum Leaflets, Harvard University. Cambridge, MA. 261 p.

Martínez H M L, A P Lachance (1979) Corn kernel hardness as an index of the alkaline cooking time for tortilla preparation. J. Food Sci. 44:377-380.

Mauricio R A S, J D C Figueroa, S Taba, M L V Reyes, F S Rincón, A G Mendoza (2004) Characterization of maize accessions by grain and tortilla quality traits. Rev. Fitotec. Mex. 27:213-222.

Moro G L, M A Lopes, J E Harbben, B R Hamaker, B A Larkins (1995) Phenotypic effects of opaque2 modifier genes in normal maize endosperm. Cereal Chem. 72:94-99.
Narváez-González E D, J D C Figueroa, S Taba, T E Castaño, R Martínez-Peniche, F S Rincón (2006a) Relationships between the microstructrure, physical features, and chemical composition of different maize accessions from Latin America. Cereal Chem. 83:595-604.

Narváez-González E D, J D C Figueroa, S Taba, F S Rincón (2006b) Kernel microstructure of Latin American races of maize and their thermal and rheological properties. Cereal Chem. 83:605-610.

Narváez-González E D, J D C Figueroa, S Taba (2007a) Aspectos microestructurales y posibles usos del maíz de acuerdo a su origen geográfico. Rev. Fitotec. Mex. 30:321-325.

Narváez-González E D, J D C Figueroa, S Taba, E T Castaño, R Martínez-Peniche (2007b) Efecto del tamaño del gránulo de almidón de maíz en sus propiedades térmicas y de pastificado. Rev. Fitotec. Mex. 30:269-277.

Norma Oficial Mexicana (2002) NMX-FF034-2002-SCFI. Productos alimenticios no industrializados para consumo humano -cereales- Parte I. Maíz blanco para proceso alcalino para tortillas de maíz y productos de maíz nixtamalizado. Especificaciones y métodos de prueba. Diario Oficial de la Federación, 22 de mayo de 2002.

Ortega-Paczka R, V R Barajas (1994) Variedades locales de maíz en el centro de Tamaulipas: Pasado y Presente. In: Memorias del $11^{\circ}$ Congreso Latinoamericano de Genética (Área Vegetal) y XV Congreso de Fitogenética. Ed. MASECA e INIFAP. Chapingo, México. $347 \mathrm{p}$.

Randolph L S (1952) New evidence of the origin of maize. Amer. Nature 86:193-202.

Romero T C, L D González, G R Reyes (2005) Geografía e historia cultural del maíz Palomero Toluqueño (Zea mays everta). Ciencia Ergo Sum. 13:47-56.

Ron J P, J J G Sánchez, A A C Jiménez, J AV Carrera, J G L Martín, M M R Morales, L L de la Cruz, S A de la P Hurtado, S M Mena, J G F Rodríguez (2006) Maíces nativos del Occidente de México I. Colectas 2004. Scientia-CUCBA 8:1-139.

Rooney L, S O Serna-Saldívar (2003) Food use of whole corn and drymilled fractions. In: Corn Chemistry and Technology. J P White, L A Johnson (eds). American Association of Cereal Chemists, Inc. $2^{\text {nd }}$ Ed . St Paul, MN. USA. pp:495-535.

Ruiz-Torres N A, F Rincón-Sánchez, L V M Hernández, J D C Figueroa, M G Loarca-Piña (2008) Determinación de compuestos fenólicos y su actividad antioxidante en granos de maíz. Rev. Fitotec. Mex. 31:29-34.

Salinas Y M, F Martínez, Gómez H J (1992) Comparación de métodos para medir la dureza del maíz (Zea mays L). Arch. Latinoam. Nutr. 42:59-63.

Salinas Y M, R J J López, B G F González, G C Vázquez (2007) Compuestos fenólicos del grano de maíz y su relación con el oscurecimiento de masa y tortilla. Agrociencia 41:295-305.

Schoch T J (1970) Food applications of corn starches. In: Corn: Culture, Processing, Products Mayor Feed and Food Crops in Agriculture and Food Series. G E Inglet (ed). The Avi Publishing Company, Inc. Westport, Connecticut. pp:195-219.

Sturtevant E L (1899) Varieties of corn. USDA Off. Exp. Stn. Bull. 571:1108.

Turrent F A, J A H Serratos, H A Mejía, C A Espinoza (2009) Liberación comercial de maíz transgénico y acumulación de transgenes en razas de maíz mexicano. Rev. Fitotec. Mex. 32:257-263.

Valdivia-Bernal R, F de J Caro-Velarde, R Medina-Torres, M Ortiz-Catón, A Espinosa-Calderón, V A Vidal-Martínez, A OrtegaCorona (2010) Contribución genética del criollo Jala en variedades eloteras de maíz. Rev. Fitotec. Mex. 33(Esp. 4):63-67.

Vázquez M G C, Márquez A R S, Márquez F S (1990) Evaluación física, química y tortillera del compuesto Pepitilla de maíz. Rev. Fitotec. Mex. 13:1-16.

Vázquez C M G, J P C Pérez, J M Hernández-Casillas, L Marrufo, E R Martínez (2010) Calidad de grano y de tortillas de maíces criollos del Altiplano y Valle del Mezquital, México. Rev. Fitotec. Mex. 33(Esp. 4):49-56.

Wellhausen E J, L M Roberts, E Hernández-X, P C Mangelsdorf (1952) Races of Maize in Mexico: Their Origin, Characteristics and Distribution. 1st ed. The Bussey Institution of Harvard University. $223 \mathrm{p}$. 